\title{
Pharmacological inhibition of S6K1 increases glucose metabolism and Akt signalling in vitro and in diet-induced obese mice
}

\author{
Michael Shum $^{1} \cdot$ Kerstin Bellmann $^{1} \cdot$ Philippe St-Pierre $^{1} \cdot$ André Marette $^{1}$
}

Received: 18 June 2015 / Accepted: 13 November 2015 / Published online: 5 January 2016

(C) Springer-Verlag Berlin Heidelberg 2016

\begin{abstract}
Aims/hypothesis The mammalian target of rapamycin complex 1 (mTORC1)/p70 ribosomal S6 kinase (S6K)1 pathway is overactivated in obesity, leading to inhibition of phosphoinositide 3-kinase (PI3K)/Akt signalling and insulin resistance. However, chronic mTORC1 inhibition by rapamycin impairs glucose homeostasis because of robust induction of liver gluconeogenesis. Here, we compared the effect of rapamycin with that of the selective S6K1 inhibitor, PF-4708671, on glucose metabolism in vitro and in vivo.

Methods We used L6 myocytes and FAO hepatocytes to explore the effect of PF-4708671 on the regulation of glucose uptake, glucose production and insulin signalling. We also treated high-fat (HF)-fed obese mice for 7 days with PF-4708671 in comparison with rapamycin to assess glucose tolerance, insulin resistance and insulin signalling in vivo. Results Chronic rapamycin treatment induced insulin resistance and impaired glucose metabolism in hepatic and muscle cells. Conversely, chronic S6K1 inhibition with PF-4708671 reduced glucose production in hepatocytes and enhanced glucose uptake in myocytes. Whereas rapamycin treatment inhibited Akt phosphorylation, PF-4708671 increased Akt phosphorylation in both cell lines. These opposite effects of the mTORC1 and S6K1
\end{abstract}

Electronic supplementary material The online version of this article (doi:10.1007/s00125-015-3839-6) contains peer-reviewed but unedited supplementary material, which is available to authorised users.

André Marette

andre.marette@criucpq.ulaval.ca

1 Department of Medicine, Quebec Heart and Lung Institute, Hôpital Laval, Pavillon Marguerite d'Youville, Room Y4308, 2705 Chemin Ste-Foy, Québec, Canada G1V 4G5 inhibitors were also observed in vivo. Indeed, while rapamycin treatment induced glucose intolerance and failed to improve Akt phosphorylation in liver and muscle of HF-fed mice, PF-4708671 treatment improved glucose tolerance and increased Akt phosphorylation in metabolic tissues of these obese mice.

Conclusions/interpretation Chronic S6K1 inhibition by PF-4708671 improves glucose homeostasis in obese mice through enhanced Akt activation in liver and muscle. Our results suggest that specific S6K1 blockade is a valid pharmacological approach to improve glucose disposal in obese diabetic individuals.

Keywords Akt - Glucose tolerance - Glucose uptake · Hepatic glucose production - Insulin resistance $\cdot$ mTORC1 . PF-4708671 · Rapamycin · S6K1 · Type 2 diabetes

$\begin{array}{ll}\text { Abbreviations } \\ \text { AMPK } & \text { AMP kinase } \\ \text { CREB } & \text { cAMP response element-binding protein } \\ \text { FOXO } & \text { Forkhead box O } \\ \text { G6Pase } & \text { Glucose-6-phosphatase } \\ \text { GTT } & \text { Glucose tolerance test } \\ \text { HF } & \text { High fat } \\ \text { HFD } & \text { High-fat diet } \\ \text { HNF4 } \alpha & \text { Hepatocyte nuclear factor 4 } \alpha \\ \text { KO } & \text { Knockout } \\ \text { MSK1 } & \text { Mitogen and stress activated kinase 1 } \\ \text { mTORC1/2 } & \text { Mammalian target of rapamycin complex 1/2 } \\ \text { PGC1 } \alpha & \text { Peroxisome proliferator-activated receptor } \gamma \\ & \text { coactivator 1 } \alpha \\ \text { PI3K } & \text { Phosphoinositide 3-kinase } \\ \text { PTT } & \text { Pyruvate tolerance test } \\ \text { RSK } & \text { p90 ribosomal S6 kinase }\end{array}$


S6K p70 ribosomal S6 kinase

TG Triacylglycerol

\section{Introduction}

Insulin is an essential hormone that regulates many physiological pathways such as cellular growth, proliferation, glucose and lipid homeostasis. Impaired insulin action has been shown to play major roles in metabolic diseases, such as obesity and type 2 diabetes. Several Ser/Thr kinases have been found to phosphorylate multiple Ser residues in IRS-1 in response to growth factors, nutrient excess or inflammatory stimuli, which inhibit insulin signalling and contribute to insulin resistance. These include the mammalian target of rapamycin (mTOR) and p70 ribosomal S6 kinase (S6K) as well as c-Jun $\mathrm{NH}_{2}$-terminal kinase (JNK) and inhibitor of nuclear factor- $\mathrm{kB}$ (IKK) [1, 2].

Both ribosomal S6 kinases-p70 ribosomal S6 kinase (S6K) and p90 ribosomal S6 kinase (RSK) - are well recognised key insulin signalling proteins $[3,4]$. The two S6K isoforms, S6K1 and S6K2, were originally thought to be redundant proteins due to their high homology. However, recent studies suggest distinct roles for each isoform (reviewed in [5]). Indeed, S6K1 participates in insulinmediated cell growth but-like mTORC1—also operates negative feedback loops at various levels of regulation. For example, several studies have shown that both mTORC1 and/or S6K1 phosphorylate specific serine residues on IRS proteins $[6,7]$. Moreover, some of these IRS-1 sites, notably Ser 1101 and Ser 636, were differentially regulated by mTOR and S6K1 $[8,9]$. We have further reported that Ser 1101 is an important target of S6K1 and that this site is hyperphosphorylated in animal models of obesity and upon nutrient excess in humans [10]. Recently, Liu et al [11] reported that $\mathrm{S} 6 \mathrm{~K} 1$ can also disrupt mTORC2 by phosphorylating Sin1 on both T86 and T398 residues. All these negative feedback control mechanisms reveal the importance of S6K1 in the regulation of insulin's pleiotropic actions.

The metabolic functions of S6K1 were also demonstrated using genetic models. Indeed, S6K1 knockout (KO) mice are resistant to high-fat (HF)-diet-induced obesity, and showed improvement of glucose tolerance and insulin signalling [12]. However, S6K1 KO mice on standard low-fat diet are also hypoinsulinaemic, glucose intolerant and have reduced beta cell mass due to a lesion in glucose sensing or insulin production [13]. Younis et al [14] further observed an improvement in glucose tolerance using antisense oligonucleotides against $\mathrm{S} 6 \mathrm{~K} 1$ in mice for 4 weeks; however, this was associated with a decrease in body weight and food intake. Interestingly, adeno-associated virus (AAV)-mediated hepatic S6K1/2 depletion was recently shown to improve systemic insulin resistance, hepatic gluconeogenesis and hepatic steatosis [15]. These studies suggest that S6K1 inhibition may be an interesting therapeutic option to alleviate obesitylinked insulin resistance and type 2 diabetes.

Acute pharmacological inhibition of the mTORC1/S6K1 pathway with rapamycin increases insulin signalling and glucose metabolism in vitro and in animal models of insulin resistance [10, 16-18]. However, chronic rapamycin treatment causes diabetes-like symptoms due to alterations in hepatic gluconeogenic genes and lipid storage in fat $[9,19,20]$. However, no studies have yet evaluated the effect of specifically inhibiting S6K1 on cellular insulin signalling, glucose metabolism and whole-body glucose homeostasis. Recently, the selective S6K1 inhibitor-PF-4708671-has been characterised [21], allowing us to test the effect of pharmacological inhibition of S6K1 on glucose metabolism for the first time. Here, we observed that PF-4708671 reduced hepatic glucose production and stimulated muscle glucose uptake in vitro, in association with increased Akt phosphorylation in both hepatocytes and myocytes. Moreover, PF-4708671 improved glucose tolerance in HF-fed obese mice by restoring Akt S473 phosphorylation in metabolic tissues, suggesting that S6K1 inhibition may represent a valid approach to improve glucose control in obese individuals with type 2 diabetes.

\section{Methods}

Reagents and antibodies Reagents and antibodies are included in Electronic Supplementary Material (ESM) Methods.

Cell culture L6 myoblasts were grown in $\alpha$-MEM (Invitrogen, Burlington, ON, Canada) supplemented with $10 \%$ (vol./vol.) FBS and differentiated into myotubes in $\alpha$-MEM with $2 \%$ (vol./vol.) FBS as previously described [22]. L6 cells were serum-deprived for $5 \mathrm{~h}$ prior to the experiments, and $100 \mathrm{nmol} / \mathrm{l}$ of insulin was used to stimulate the cells during the last $5 \mathrm{~min}$ of deprivation. FAO hepatocytes were maintained in RPMI media (Invitrogen), serum-deprived overnight and stimulated with the indicated concentration of insulin. Vehicle (dimethyl sulfoxide, DMSO $0.02 \%$ [vol./vol.]) or rapamycin $(25 \mathrm{nmol} / \mathrm{l})$ or PF-4708671 $(10 \mu \mathrm{mol} / \mathrm{l})$ were used since p70 S6 kinase is fully inhibited at this dose [21]. Only mycoplasma free cells were used.

Western analyses Western blots were performed as described [19]. Briefly, equal amounts of proteins were separated by SDS-PAGE (7.5\% [wt/vol.]) and transferred onto nitrocellulose membrane. Membranes were blocked in 5\% (wt/vol.) fish gelatin diluted in Tris $\mathrm{pH} 7.4+0.1 \%$ (vol./vol.) Tween (TBS-T) and incubated overnight at $4^{\circ} \mathrm{C}$ with the respective antibodies diluted in $1 \%(\mathrm{wt} / \mathrm{vol}$.) fish gelatin in TBS-T. 
Glucose uptake Glucose (2-DG) uptake was performed as previously described [22] (see ESM Methods).

Glucose production Glucose production was evaluated in FAO hepatocytes as described previously [23] (see ESM Methods).

Animal studies Animal handling and treatment were approved by the Animal Care and Handling Committee of Laval University. Male C57B1/6 mice (6 weeks old) were purchased from Charles River Laboratories (St Constant, QC, Canada) and housed individually in cages in a room kept at $23 \pm 1^{\circ} \mathrm{C}$ with a $12 \mathrm{~h} \mathrm{light} / 12 \mathrm{~h}$ dark cycle. Mice were randomly distributed among groups but matched for body weight at the beginning of the studies. The glucose and pyruvate tolerance tests were done blinded. Male C57Bl/6 mice (6 weeks old) were fed high-fat diet (HFD, 60\% energy from fat, Research Diets, D12492 (New Brunswick, NJ, USA)) for 12 weeks before being randomly assigned to three groups: (1) control (HF) receiving vehicle (8\% EtOH [vol./vol.], $0.2 \%$ [wt/vol.] carboxymethylcellulose sterile); (2) treated with PF-4708671 (35 mg kg ${ }^{-1} \mathrm{day}^{-1}$, i.p.); or (3) treated with rapamycin ( $2 \mathrm{mg} \mathrm{kg}^{-1} \mathrm{day}^{-1}$, i.p.) for 7 days while being kept on the same HFD (ESM Methods).

i.p. glucose and pyruvate tolerance tests After 12 weeks of HFD feeding, mice were fasted for $6 \mathrm{~h}$ and injected i.p. with glucose $(1 \mathrm{~g} / \mathrm{kg})$ diluted in saline, $0.9 \%$ (wt/vol. $)(\mathrm{NaCl}$ $154 \mathrm{mmol} / \mathrm{l}$ ). For the pyruvate tolerance test (PTT), mice were fasted for $6 \mathrm{~h}$ and injected i.p. with pyruvate ( $2 \mathrm{~g} / \mathrm{kg}$; Sigma-Aldrich, Oakville, ON, Canada) diluted in saline, $0.9 \%$ (wt/vol.).

Plasma/tissue determinations Blood glucose levels were measured by a One Touch Mini Ultra Glucometer (LifeScan, CA, USA). Plasma insulin was determined by radioimmunoassay (Linco Research, St Charles, MO, USA). Triacylglycerol (TG) levels in plasma and liver were measured by enzymatic methods according to the manufacturers' instructions (Randox Lab kit, WV, USA). Total lipid was isolated from $0.1 \mathrm{~g}$ frozen liver using chloroform-methanol extraction [24]. Hepatic TG content was enzymatically determined from reconstituted lipid extract. Liver glycogen was determined using the phenolsulphuric acid reaction [25].

Nuclear extracts Liver nuclear extracts were prepared as previously described [19] and subjected to immunoblotting as described above.

RNA extraction and quantitative PCR analysis RNA extraction and quantitative PCR analysis were performed as described previously [19] (see ESM Methods).
Quantification and statistical analyses One- and two-way ANOVA with Newman-Keuls post hoc was performed with Sigma Plot version 12 (Systat Software, San Jose, CA, USA); $p$ values were considered significant if they were $<0.05$. SEMs are represented in the graphs. The criterion for exclusion of animals was a $>20 \%$ deviation from the mean weight of the other animals of the same group. The criterion for the exclusion of any data point was a distance $> \pm 2.5 \times$ SD from the mean of the other data from the same group.

\section{Results}

\section{S6K1 inhibition decreases S6 phosphorylation} and increases Akt phosphorylation in hepatocytes

As the main target of S6K1, ribosomal S6 protein phosphorylation was first measured after treatment with PF-4708671 in hepatocytes. Both basal and insulin-stimulated S6 phosphorylation on S235/6 were rapidly inhibited by PF-4708671, and the inhibitory effect was maintained for up to $24 \mathrm{~h}$ in insulintreated cells (Fig. 1). Moreover, S6K1 Thr389 phosphorylation increased after PF-4708671 treatment, a finding that is in line with previous reports, and that was suggested to involve some feedback pathways by which S6K1 might regulate its own phosphorylation by mTORC1 $[21,26]$. As previously shown using genetic tools in cells [10], pharmacological S6K1 inhibition by PF-4708671 reduced insulin-stimulated IRS1 S1101 phosphorylation. PF-4708671 treatment increased insulin-induced Akt phosphorylation from $5 \mathrm{~h}$ and this effect was sustained for up to $24 \mathrm{~h}$ (Fig. 1b, c). We also ruled out that PF-4708671 might inhibit mitogen and stress activated kinase 1 (MSK1) since this inhibitor did not inhibit Thr581 MSK1 and Ser133 cAMP response element-binding protein (CREB) induced by phorbol myristate acetate (PMA) in hepatocytes (ESM Fig. 1), in line with previous work by Pearce et al [21].

S6K1 inhibition decreases glucose production in hepatic cells To evaluate the role of S6K1 in liver glucose metabolism, we measured basal and insulin-suppressed glucose production in FAO hepatic cells. PF-4708671 treatment for $5 \mathrm{~h}$ decreased basal glucose production, which was further reduced with increasing concentrations of insulin (Fig. 2a). We previously reported that chronic rapamycin treatment increased hepatic glucose production in rats and FAO cells [19]. We thus compared the effect of chronic PF-4708671 treatment with that of rapamycin by treating FAO cells for $72 \mathrm{~h}$ with either drug before measuring glucose production. As illustrated in Fig. 2b, chronic S6K1 inhibition reduced basal glucose production while chronic rapamycin treatment increased it. When corrected for differences in basal rates of glucose production, the results revealed that rapamycin treatment reduced the 
Fig. 1 Time-dependent S6K1 inhibition by PF-4708671. Insulin signalling analysis after timedependant S6K1 inhibition by PF-4708671 (a, b). FAO cells were treated with $10 \mu \mathrm{mol} / \mathrm{l} \mathrm{PF}$ 4708671 for the indicated times followed by $5 \mathrm{~min}$ insulin treatment $(100 \mathrm{nmol} / \mathrm{l})$. These blots are representative of at least three separate experiments. (c) Quantification of basal (white bars) and insulin-stimulated (black bars) phospho-Akt are shown; $n=5$. ${ }^{*} p<0.05$, vehicle vs PF-4708671 treated cells a

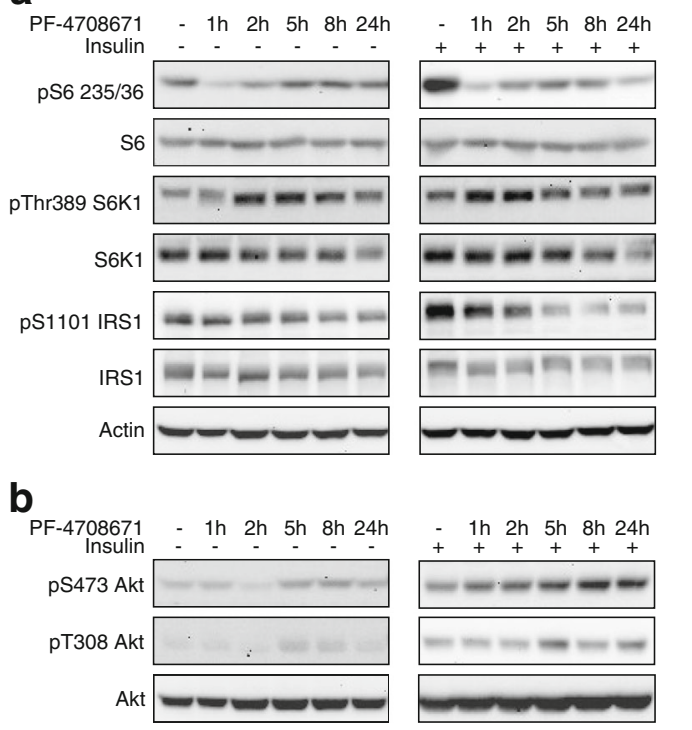

C
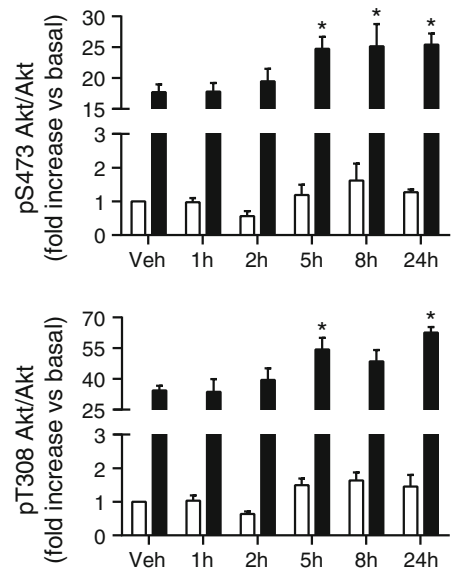

suppressing effect of insulin while insulin action on glucose production remained unaffected by S6K1 inhibition (Fig. 2c, d). Nuclear extractions of proteins involved in gluconeogenic enzyme expression revealed that hepatocyte nuclear factor $4 \alpha$ (HNF4 $\alpha$ ), forkhead box O (FOXO) and CREB were decreased after $72 \mathrm{~h}$ inhibition of S6K1 while chronic rapamycin treatment increased the levels of these proteins compared with vehicle (Fig. 2e). However, peroxisome proliferator-activated receptor $\gamma$, coactivator $1 \alpha(\mathrm{PGC} 1 \alpha)$ expression in the nucleus was not affected by the treatments. In line with these results, FAO cells treated with PF-4708671 showed reduced mRNA expression of the gluconeogenic enzymes PEPCK (also known as Pck1) and G6Pase (also known as G6pc) (Fig. 2f). Chronic rapamycin treatment increased G6Pase protein content but PF-4708671 failed to affect glucose-6-phosphate (G6Pase) and PEPCK protein levels (Fig. 2g). As expected, both acute and chronic PF-4708671 treatment reduced insulin-mediated S6 phosphorylation (Fig. 2h). On the other hand, chronic rapamycin treatment completely reduced both basal and insulin-stimulated S6 phosphorylation, even below the level of vehicle-treated control cells. Interestingly, acute and chronic inhibition of S6K1 increased insulin-induced Akt phosphorylation while this was fully abrogated in cells chronically exposed to rapamycin (Fig. 2h). Whereas rapamycin treatment increased basal Akt T308 phosphorylation, insulin-induced Akt S473 phosphorylation was disrupted by the inhibitor.

S6K1 inhibition increases glucose uptake in L6 myocytes We next determined whether S6K1 inhibition improves glucose uptake using L6 myocytes. PF-4708671 markedly increased basal glucose uptake after $5 \mathrm{~h}$ and this effect persisted up to $48 \mathrm{~h}$ compared with vehicle-treated cells (Fig. 3a). Insulin did not further increase the effect of the S6K1 inhibitor on glucose uptake. Conversely, $48 \mathrm{~h}$ rapamycin treatment significantly reduced basal and insulin-mediated glucose uptake in these muscle cells. The stimulatory effect of PF-4708671 on glucose uptake was fully abolished by Akt inhibition using the Akt inhibitor, Viii (Fig. 3b). As in hepatocytes, PF-4708671 reduced insulin-induced S6 phosphorylation while chronic rapamycin treatment fully abrogated this response in L6 muscle cells (Fig. 3c). After $48 \mathrm{~h}$ treatment, S6K1 inhibition increased Akt phosphorylation. However, rapamycin was found to decrease Akt S473 while increasing both basal and insulinmediated Akt T308 phosphorylation (Fig. 3c). Recently, PF-4708671 was reported to enhance AMP kinase (AMPK) phosphorylation in mouse embryonic fibroblasts lacking S6K [27]. However, AMPK Thr172 phosphorylation was not increased after 5 and $72 \mathrm{~h}$ treatment of L6 myocytes with the S6K1 inhibitor (Fig. 3d). Collectively, these results demonstrate that glucose metabolism is increased after PF-4708671 exposure in both muscle and hepatic cells.

In vivo treatment with PF-4708671 improves glucose homeostasis in obese mice To evaluate the therapeutic potential of PF-4708671 in vivo, we treated HFD-fed obese mice with PF-4708671 for 1 week and compared its effect with that of rapamycin. As expected, the HFD induced body weight gain, adiposity, hyperglycaemia and hyperinsulinemia as compared with mice fed the chow diet (Table 1). While PF-4708671 did not affect body weight or adiposity, rapamycin treatment was found to significantly reduce body weight, which was partly related to decreased inguinal adipose tissue weight. Interestingly, only 1 week of PF-4708671 treatment was found to improve fasting glucose whereas rapamycin further increased fasting hyperglycaemia in obese mice. In addition to fasting glucose, rapamycin treatment was also found to increase fasting insulinaemia as previously reported in various rodent models [19, 20, 28, 29]. 
a

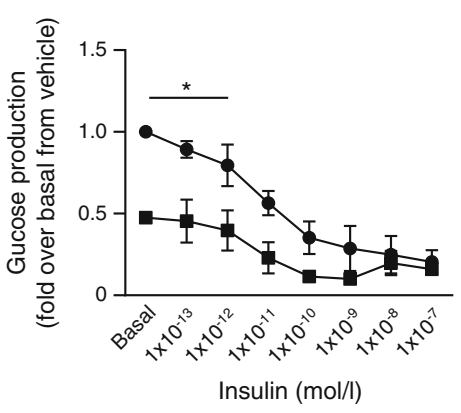

C

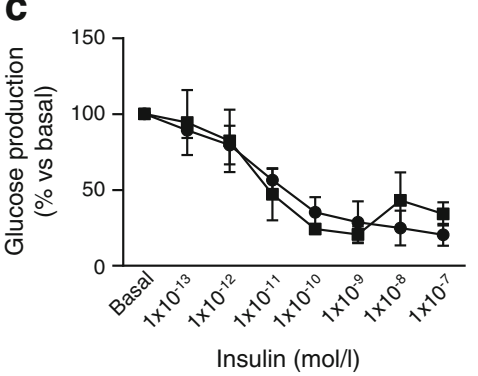

g
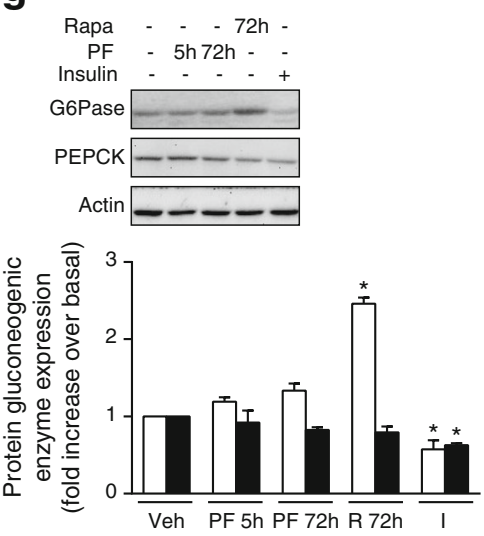

b

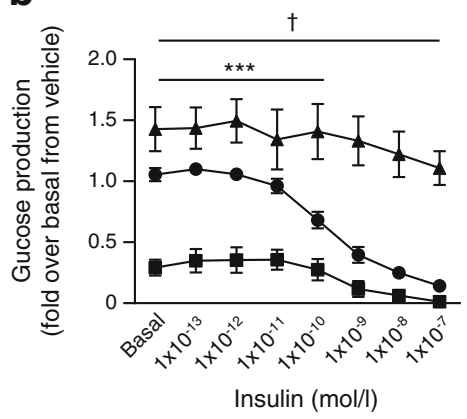

d

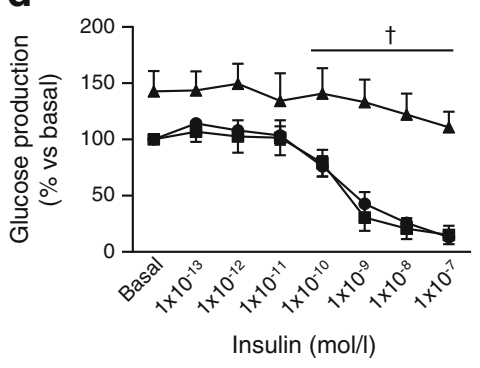

h

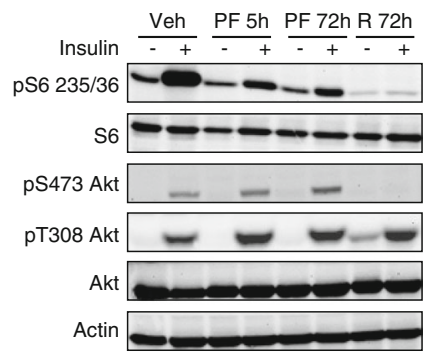

e

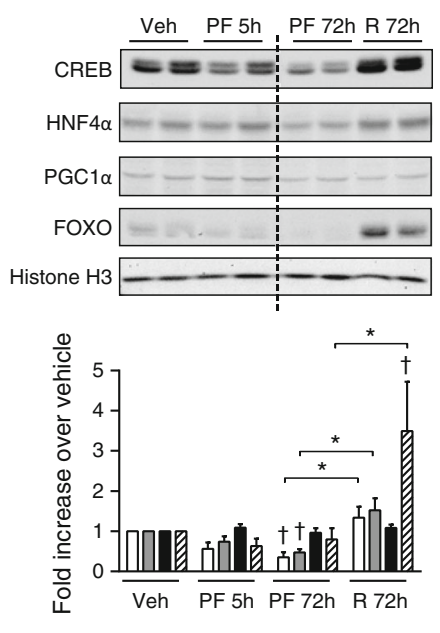

f
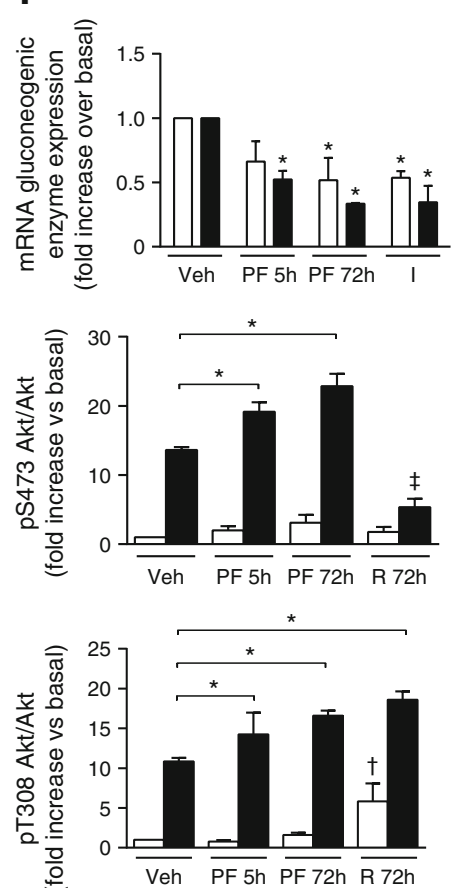

Fig. 2 S6K1 inhibition decreased glucose production and increased Akt phosphorylation in hepatocytes. Glucose production was evaluated in FAO cells as described in Methods. Cells were treated with vehicle (black circles) or with $10 \mu \mathrm{mol} / 1 \mathrm{PF}-4708671$ (black squares) for $5 \mathrm{~h}(\mathbf{a}, \mathbf{c})$ or $72 \mathrm{~h}(\mathbf{b}, \mathbf{d})$ and compared with cells treated with $25 \mathrm{nmol} / 1$ rapamycin (black triangles) for $72 \mathrm{~h} ; n=3-5 ;{ }^{\dagger} p<0.05$ for rapamycin- vs vehicleand PF-4708671-treated cells; ${ }^{*} p<0.05, * * * p<0.001$ for PF-4708671 vs vehicle. (e) Representative blots and quantification of CREB (white bars), HNF $4 \alpha$ (grey bars), PGC1 $\alpha$ (black bars), FOXO (hatched bars) and histone $\mathrm{H} 3$ of nuclear extracts from FAO cells treated with $10 \mu \mathrm{mol} / 1$ $\mathrm{PF}-4708671$ or $25 \mathrm{nmol} / 1$ rapamycin. ${ }^{\dagger} p<0.05$ vs vehicle from the same treatment group. (f) mRNA levels of G6pase (white bars) and Pepck (black bars) were assessed by semi-quantitative RT-PCR in FAO cells treated with PF-4708671 or insulin (100 nmol/1, 24 h). The graphs depict mRNA expression in FAO cells of target genes corrected for the expression of $36 B 4$ (also known as Rplp0) as a control gene, $n=3,{ }^{*} p<0.05$ vs vehicle. (g) Protein levels of G6Pase (white bars) and PEPCK (black bars) were measured in FAO cells treated with PF-4708671, rapamycin or insulin (100 nmol/l, 24 h), $n=3,{ }^{*} p<0.05$ vs vehicle. (h) Phospho-S6 235/36 and Akt phosphorylation levels were measured in FAO cells treated with $10 \mu \mathrm{mol} / \mathrm{l} \mathrm{PF}-4708671$ or $25 \mathrm{nmol} / \mathrm{l}$ rapamycin and basal (white bars) or $5 \mathrm{~min}$ insulin-stimulated cells (black bars) $(100 \mathrm{nmol} / \mathrm{l})$ were analysed. ${ }^{\star} p<0.05$ vs insulin- and PF-insulin-treated groups, ${ }^{*} p<0.05,{ }^{\dagger} p<0.05$ vs vehicle treated cells, as indicated. I, insulin; PF, PF-4708671; R, rapamycin; Veh, vehicle

partially reversed by S6K1 inhibition but further exacerbated by rapamycin treatment (Fig. 4a, b). The improvement of glucose tolerance by S6K1 inhibition was independent of 
a

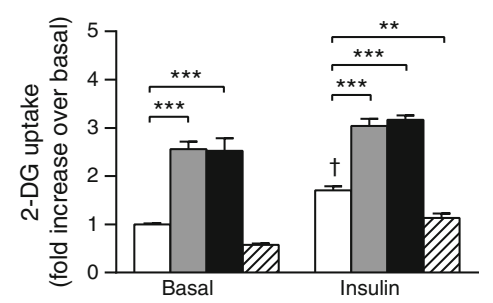

b

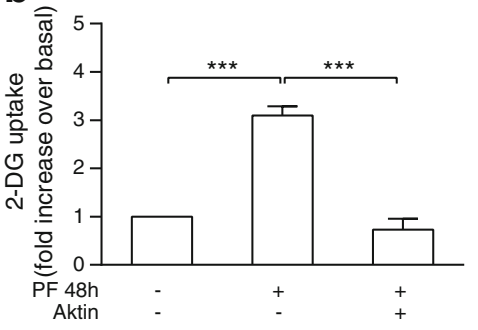

C
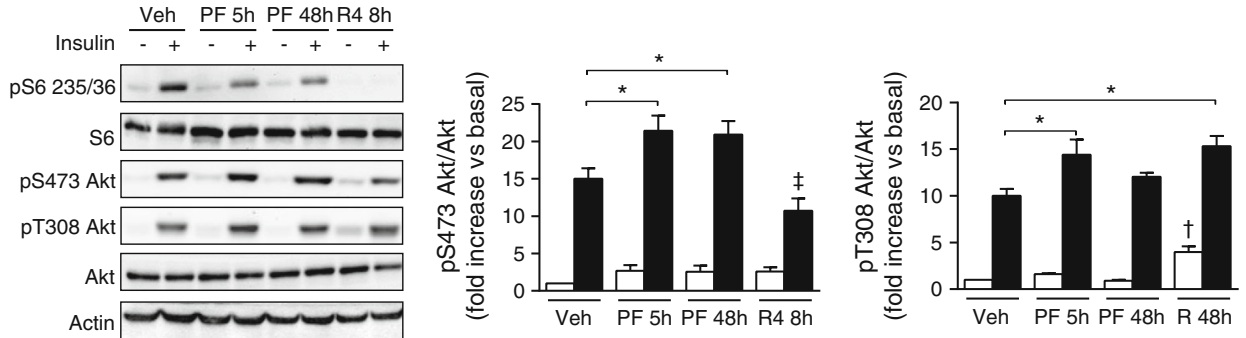

d

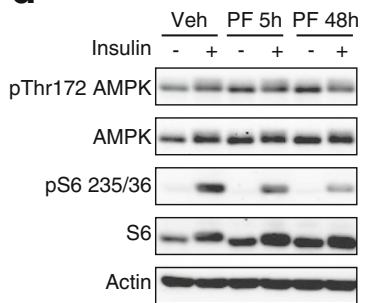

Fig. 3 S6K1 inhibition increased glucose uptake and increased Akt phosphorylation in L6 myotubes. (a) Glucose (2-DG) uptake was evaluated in L6 cells treated with vehicle (white bars) or $10 \mu \mathrm{mol} / 1$ PF-4708671 (5 h, grey bars; $48 \mathrm{~h}$, black bars) or $25 \mathrm{nmol} / 1$ rapamycin for $48 \mathrm{~h}$ (hatched bars) as described in the Methods. Results are presented as fold over basal conditions and are the means \pm SEM of three independent experiments. ${ }^{\dagger} p<0.05$ for insulin vs basal conditions (white bars). (b) Cells were used as in (a) with or without Akt inhibitor-Viii (Akt i; $10 \mu \mathrm{mol} / \mathrm{l}$ ) for the last hour. (c) Phospho-S6 235/36 and Akt phosphorylation levels were measured in L6 cells treated with $10 \mu \mathrm{mol} / 1$

changes in plasma insulin levels while rapamycin increased the insulin response during the GTT (Fig. 4c). HF feeding also increased liver TG but decreased liver glycogen content (Fig. 4d, e). PF-4708671 tended to decrease slightly liver TG and glycogen content compared with the HF group while rapamycin tended to increase them leading to a statistically significant difference between the drugs.

\section{Chronic PF-4708671 and rapamycin effect on gluconeo-} genesis in vivo The rapamycin-induced deterioration of glucose tolerance was also associated with increased gluconeogenesis as measured by a PTT, which was not affected by PF4798671 treatment (Fig. 5a). Accordingly, PF-4798671-treated mice expressed a similar level of nuclear PGC $1 \alpha, \mathrm{HNF} 4 \alpha$ and CREB compared with HF-fed control mice (Fig. 5b). As expected from the increased

PF-4708671 or $25 \mathrm{nmol} / 1$ rapamycin and basal (white bars) or $5 \mathrm{~min}$ insulin-stimulated cells (black bars) $(100 \mathrm{nmol} / \mathrm{l})$ were analysed, $n=$ $4-5 .{ }^{\star} p<0.05$ vs insulin- and PF-insulin-treated groups (black bars), ${ }^{\dagger} p<0.05$ vs vehicle treated cells. (d) Phospho-Thr172 AMPK and phospho-S6 235/36 levels were measured in L6 cells pre-treated with $10 \mu \mathrm{mol} / 1 \mathrm{PF}-4708671$ or vehicle (DMSO $0.02 \%$ ) and basal (white bars) or $5 \mathrm{~min}$ insulin-stimulated cells (black bars) $(100 \mathrm{nmol} / \mathrm{l})$ were analysed, $n=3 .{ }^{*} p<0.05,{ }^{* *} p<0.01,{ }^{* * *} p<0.001$ in all figure parts, as indicated. AMPK, AMP kinase; PF, PF-4708671; R/Rapa, rapamycin; Veh, vehicle

hepatic gluconeogenesis in the rapamycin-treated mice, we found higher nuclear expression of CREB and $\mathrm{HNF} 4 \alpha$ in the liver of these animals. HF-fed mice treated with the S6K1 inhibitor expressed similar mRNA levels of gluconeogenic enzymes compared with vehicle-treated HF-fed controls while G6Pase mRNA levels were increased in liver of rapamycin-treated mice (Fig. 5c).

Chronic PF-4708671 improved Akt S473 phosphorylation in HF-fed mice tissues To further explore the mechanisms underlying the metabolic phenotype of PF-4708671-treated mice, we next analysed Akt phosphorylation in liver, muscle and adipose tissues. In liver, the HFD induced a decrease in insulin-stimulated Akt S473 phosphorylation in all tissues, which was fully restored by PF-4708671 in liver and muscle, but only partially improved in adipose tissue. In contrast, 
Table 1 Effect of 7-day PF-4708671 and rapamycin treatment on weight, food intake and metabolic variables of treated mice

\begin{tabular}{lcccc}
\hline Metabolic variable & Standard diet & HFD & \\
\cline { 2 - 4 } & Vehicle & Vehicle & PF & Rapa \\
\hline Body weight (g) & $28.78 \pm 0.33^{\mathrm{a}}$ & $39.21 \pm 1.23^{\mathrm{b}}$ & $38.75 \pm 0.74^{\mathrm{b}, \mathrm{c}}$ & $35.51 \pm 0.90^{\mathrm{c}}$ \\
Energy intake (kJ/day) & $45.63 \pm 1.08$ & $45.93 \pm 1.51$ & $41.49 \pm 0.96$ & $44.34 \pm 1.51$ \\
Fasting glucose (mmol/) & $8.80 \pm 0.26^{\mathrm{a}}$ & $10.46 \pm 0.54^{\mathrm{b}}$ & $9.18 \pm 0.26^{\mathrm{a}}$ & $12.83 \pm 0.45^{\mathrm{c}}$ \\
Fasting insulin (pmol/1) & $103.26 \pm 10.33^{\mathrm{a}}$ & $368.29 \pm 34.42^{\mathrm{b}}$ & $411.32 \pm 41.30^{\mathrm{b}}$ & $619.56 \pm 58.51^{\mathrm{c}}$ \\
TG (mmol/l) & $0.50 \pm 0.06^{\mathrm{a}}$ & $0.56 \pm 0.04^{\mathrm{a}, \mathrm{b}}$ & $0.45 \pm 0.05^{\mathrm{a}, \mathrm{b}}$ & $0.60 \pm 0.04^{\mathrm{b}}$ \\
Liver (g) & $1.30 \pm 0.03$ & $1.18 \pm 0.08$ & $1.20 \pm 0.03$ & $1.15 \pm 0.04$ \\
Pancreas (g) & $0.22 \pm 0.02$ & $0.19 \pm 0.02$ & $0.22 \pm 0.02$ & $0.19 \pm 0.01$ \\
Gastrocnemius (g) & $0.33 \pm 0.01$ & $0.32 \pm 0.01$ & $0.32 \pm 0.01$ & $0.33 \pm 0.01$ \\
Heart (g) & $0.15 \pm 0.01^{\mathrm{a}}$ & $0.13 \pm 0.01^{\mathrm{a}, \mathrm{b}}$ & $0.13 \pm 0.01^{\mathrm{a}, \mathrm{b}}$ & $0.12 \pm 0.01^{\mathrm{b}}$ \\
Inguinal WAT (g) & $0.24 \pm 0.02^{\mathrm{a}}$ & $1.22 \pm 0.15^{\mathrm{b}, \mathrm{c}}$ & $1.47 \pm 0.13^{\mathrm{b}}$ & $0.99 \pm 0.10^{\mathrm{c}}$ \\
Ret WAT (g) & $0.11 \pm 0.02^{\mathrm{a}}$ & $0.73 \pm 0.05^{\mathrm{b}}$ & $0.68 \pm 0.04^{\mathrm{b}}$ & $0.62 \pm 0.54^{\mathrm{b}}$ \\
Epididymal WAT (g) & $0.42 \pm 0.03^{\mathrm{a}}$ & $2.11 \pm 0.10^{\mathrm{b}}$ & $2.10 \pm 0.09^{\mathrm{b}}$ & $1.90 \pm 0.09^{\mathrm{b}}$ \\
BAT (g) & $0.09 \pm 0.01$ & $0.12 \pm 0.01$ & $0.12 \pm 0.01$ & $0.10 \pm 0.01$ \\
Fat mass (\%) & $2.41 \pm 0.06^{\mathrm{a}}$ & $11.99 \pm 1.47^{\mathrm{b}}$ & $11.05 \pm 0.78^{\mathrm{b}}$ & $11.83 \pm 0.93^{\mathrm{b}}$ \\
Lean mass (g) & $22.64 \pm 0.55$ & $22.34 \pm 0.73$ & $22.81 \pm 0.51$ & $21.16 \pm 0.33$ \\
\hline
\end{tabular}

Data are means \pm SE ( $n=7-10$ control and HFD group; $n=8-14$ for PF-4708671 and rapamycin group)

Means not sharing a common superscript are significantly different from each other, $p<0.05$

BAT, brown adipose tissue; PF, PF-4708671; Rapa, rapamycin; Ret, retroperitoneal; WAT, white adipose tissue rapamycin treatment only partially restored Akt S473 phosphorylation in liver but not in muscle and adipose tissues (Fig. 6a-c). The effect of the drug on Akt Thr308 phosphorylation was less clear. Whereas both PF-4708671 and rapamycin treatments tended to improve insulin-induced
Thr308 phosphorylation in liver and muscle (Fig. 6a, b), only rapamycin was found to improve the phosphorylation of this site in adipose tissue of obese mice (Fig. 6c). Both PF-4708671 and rapamycin were found to blunt the HFD-induced increased S1101 phosphorylation of IRS- a

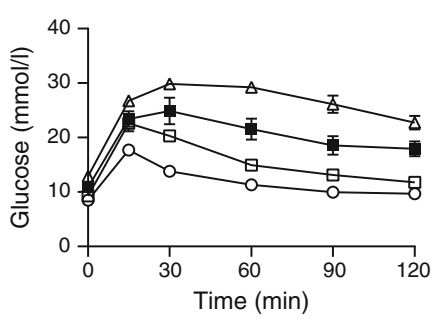

d

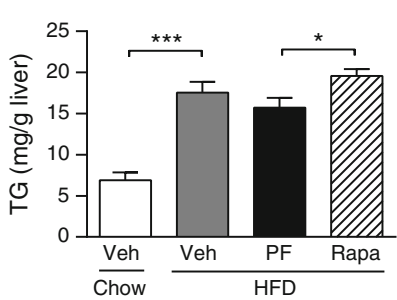

b
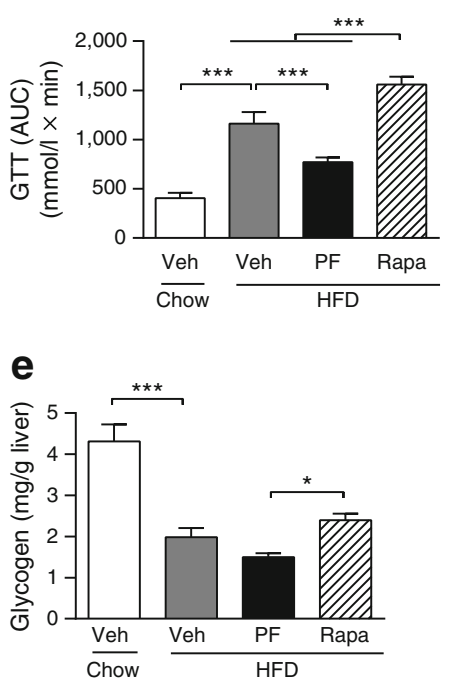

C

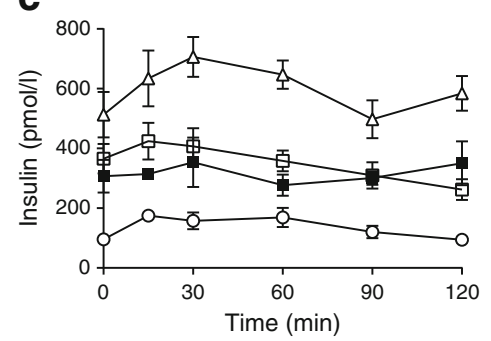

Fig. 4 S6K1 inhibition improved glucose tolerance while rapamycin exacerbated glucose intolerance in HF-fed mice. Mice were treated with PF-4708671 and rapamycin as described in the Methods and fasted for $6 \mathrm{~h}$ before i.p. GTTs. (a) Plasma glucose, (b) AUC of plasma glucose and (c) insulin levels were measured during a GTT. Chow-fed mice (white circles and white bars), and HF-fed mice treated with vehicle (black squares and grey bars), PF-4708671 (white squares and black bars) and rapamycin (white triangles and hatched bars), $n=6-8$ for each group. (d) Liver TG and (e) liver glycogen content were measured as described in the Methods. $n=6-8$ for each group. ${ }^{*} p<0.05, * * * p<0.001$. PF, PF-4708671; Rapa, rapamycin; Veh, vehicle 


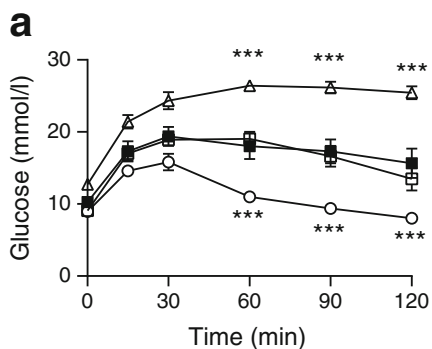

b
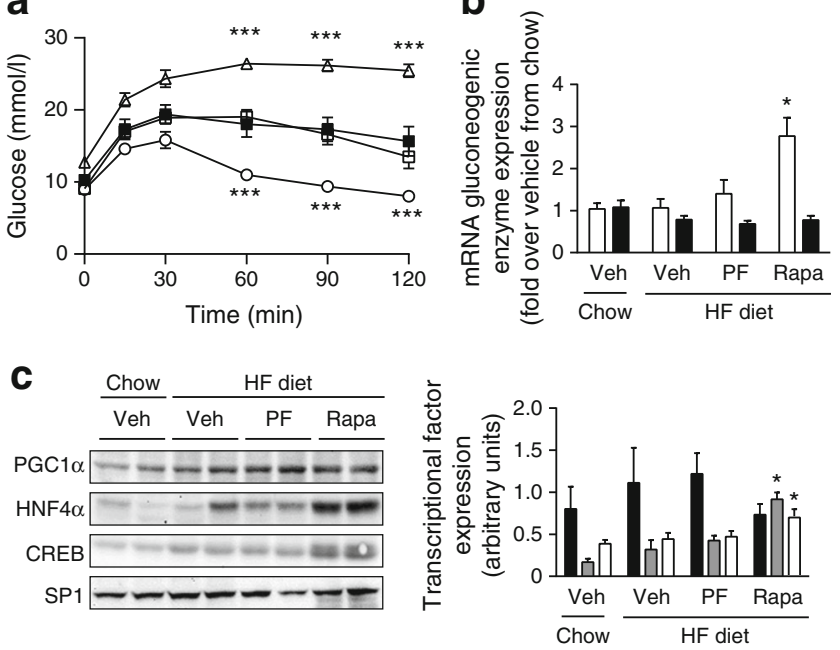

Fig. 5 PF-4708671 did not reproduce the deleterious effect of chronic rapamycin on hepatic gluconeogenesis. (a) Plasma glucose levels were measured during an i.p. PTT. $n=6-8$ for each group. Chow-fed mice (white circles), HF-fed mice (black squares), HF-fed mice treated with PF-4708671 (white squares) and HF-fed mice treated with rapamycin (white triangles). (b) mRNA levels of G6pase (white bars) and Pepck (black bars) were assessed by semi-quantitative RT-PCR; $n=6-8$. (c) Representative blots and densitometric analysis of normalisation of total protein/SP1 protein of PGC1 $\alpha$ (black bars), HNF $4 \alpha$ (grey bars), CREB (white bars) and SP1 proteins in nuclear extracts prepared from liver samples. $n=3-4$ for each group. ${ }^{*} p<0.05,{ }^{* * *} p<0.001$, HF group treated with vehicle vs PF-4708671 and rapamycin-treated cells. PF, PF-4708671; Rapa, rapamycin; Veh, vehicle

1 in liver and adipose tissue (ESM Fig. 2). IRS-1 phosphorylation on S1101 could not be detected in muscle (data not shown).

To determine whether the tissue-specific effects of PF-4708671 on Akt phosphorylation may be related to differential inhibition of S6K1 in liver, muscle and adipose tissue, we further evaluated the phosphorylation state of S6 in those tissues. Unexpectedly, PF-4708671 did not inhibit S6 phosphorylation, which was, however, completely inhibited by rapamycin under both basal and insulinstimulated conditions (Fig. 7a-c). On the other hand, treatment with PF-4708671, but not rapamycin, caused a marked increase in S6K1 phosphorylation, at least in liver and adipose tissue (Fig. $7 \mathrm{a}-\mathrm{c}$ ). This is consistent with the inhibition of S6K1 by PF-4708671, as seen in vitro (Fig. 1) and previous reports $[21,26]$, suggesting that other kinases might phosphorylate ribosomal S6 proteins in those tissues in vivo.

\section{Discussion}

The mTORC1/S6K pathway regulates many essential processes such as growth, proliferation, protein synthesis and lipogenesis. In addition, several studies have emphasised the role of mTORC1/S6K1 in metabolism since this pathway senses nutrients and energy (ATP) but also regulates insulin signalling at various levels. Acute treatment $(<2 \mathrm{~h})$ with the mTORC1 inhibitor, rapamycin, improves the metabolic actions of insulin through disrupting the negative feedback loop towards phosphoinositide 3-kinase (PI3K)/Akt signalling in both cellular and animal models [10,16-18]. However, when administered chronically $(>24 \mathrm{~h})$ rapamycin also has important adverse metabolic effects in rodent models and in humans, causing hyperlipidaemia, hyperglycaemia and hyperinsulinaemia $[9,19,30,31]$.

In the present study, we show that chronic S6K1 inhibition does not reproduce the adverse effects observed after chronic rapamycin treatment. In vitro studies using hepatic and muscle cells revealed that S6K1 inhibition with PF-4708671 reduced hepatic glucose production and increased muscle glucose uptake, and these effects were seen even in the absence of insulin stimulation. In both cell types we found that, in contrast to rapamycin treatment, PF-4708671 increased insulin-induced Akt phosphorylation. The therapeutic relevance of these cellular findings was further demonstrated in HF-fed mice, in which chronic treatment with PF-4708671 for 7 days was found to improve glucose tolerance, while chronic rapamycin exacerbated glucose intolerance in these obese animals. This improvement in glucose homeostasis after S6K1 inhibition was associated with a restoration of Akt phosphorylation in liver, muscle and adipose tissues. Although PF-4708671 did not inhibit MSK1 (ESM Fig. 1) or RSK1 [23] in muscle and hepatic cells, we cannot completely exclude that part of its beneficial effects on glucose homeostasis in vivo is explained by partial inhibition of these enzymes. However, our finding that pharmacological inhibition of S6K1 improves glucose metabolism is in line with previous studies using genetic models of S6K1 deficiency. Um et al [12] first showed that $\mathrm{S} 6 \mathrm{~K}^{-/-}$mice are protected against diet-induced obesity and insulin resistance, and this was associated with increased Akt S473 phosphorylation in insulin target tissues. More recent studies have also shown that antisense oligonucleotides against S6K1 in rats or liver-specific downregulation of S6K1/2 using a lentiviral approach in the liver of HF-fed mice improved glucose tolerance and insulin sensitivity, which was associated with increased Akt S473 phosphorylation in the liver and adipose tissue in the latter study $[14,15]$.

Our results show that S6K1 inhibition represents a more suitable target to improve glucose homeostasis as compared with abrogating the mTORC1/S6K1 pathway with rapamycin, which has been reported to impair glucose and lipid metabolism after chronic in vivo exposure [9, 19, 29]. The adverse effects of chronic rapamycin treatment are partly due to off-target effects on mTORC2 [29, 32, 33], which impairs Akt $\mathrm{S} 473$ phosphorylation and activity $[34,35]$, but these side effects were not observed with selective S6K1 inhibition in the present study. In contrast, we found that 

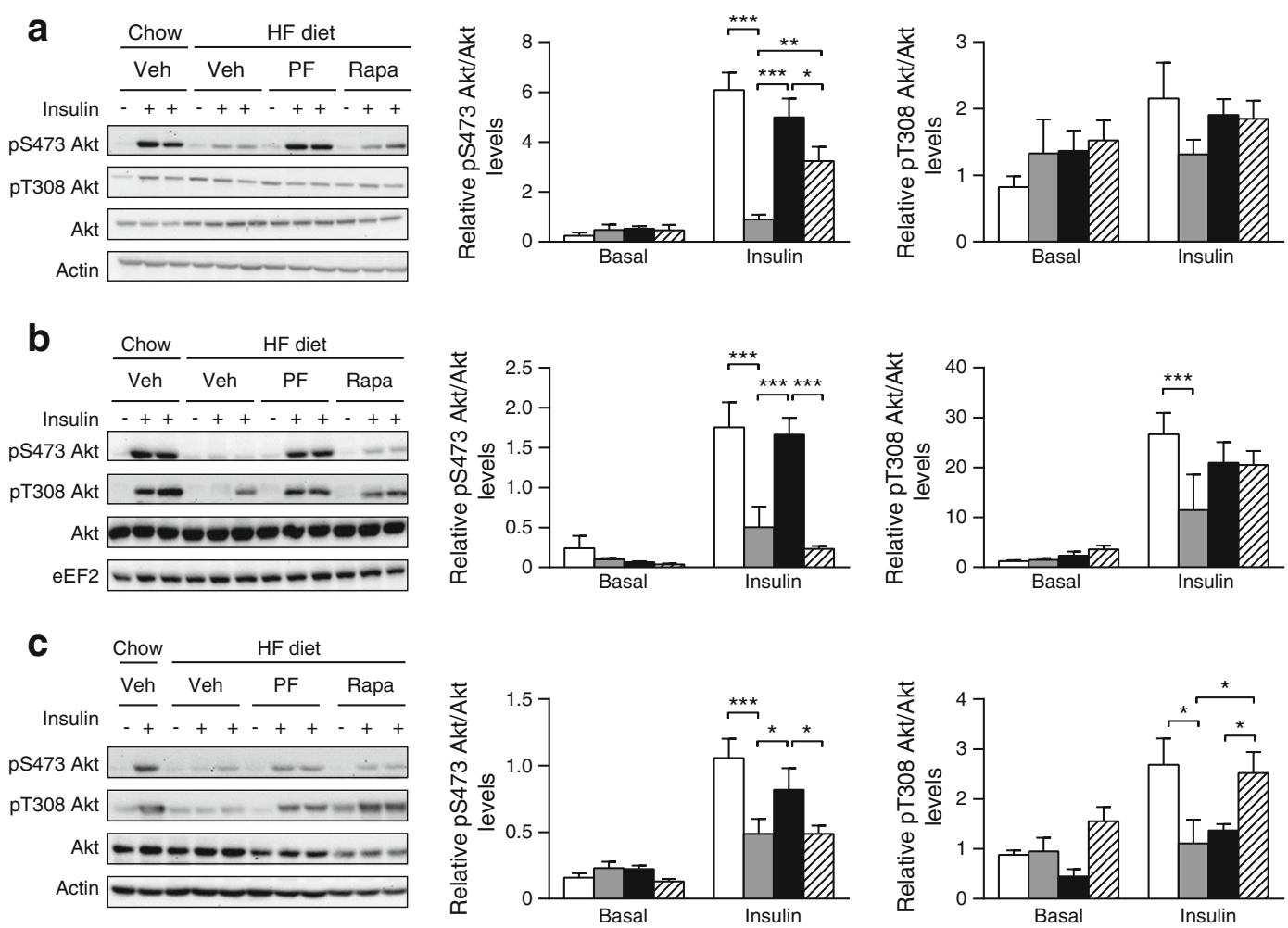

Fig. 6 S6K1 inhibition increased Akt phosphorylation. Chow-fed mice (white bars), HF-fed mice treated with vehicle (grey bars), HF-fed mice treated with PF-4708671 (black bars) and HF-fed mice treated with rapamycin (hatched bars) were fasted for $6 \mathrm{~h}$ before saline or insulin (3.8 U/kg caudal, $5 \mathrm{~min}$ ) vein injection and tissues were analysed for Akt phosphorylation. Liver (a), gastrocnemius muscle (b) and epididymal white adipose tissue (c) were lysed and equal amounts of proteins were separated on SDS-PAGE and processed for western blot analyses using the indicated antibodies. $n=5-8$ for each group. ${ }^{*} p<0.05$, ${ }^{* *} p<0.01,{ }^{* * *} p<0.001$. PF, PF-4708671; Rapa, rapamycin; Veh, vehicle

PF-4708671 robustly increased basal glucose uptake in L6 myocytes and insulin did not further increase the stimulatory effect of PF-4708671 on this process. This insulinindependent effect of the S6K1 inhibitor is consistent with the finding that S6K-mediated Sin 1 phosphorylation negatively regulates $\mathrm{mTORC} 2$ independently from insulin signalling [11].

S6K1 inhibition in hepatic cells was associated with increased Akt S473 phosphorylation and lower mRNA expression of PEPCK and G6Pase gluconeogenic enzymes as well as a reduction in CREB, FOXO and HFN $4 \alpha$ nuclear levels. However, PF-4708671 failed to significantly reduce PEPCK or G6Pase protein expression despite lower mRNA expression of these enzymes. It is possible that G6Pase and PEPCK proteins are only reduced in specific cellular compartments [38-40]. Indeed, we have used a PEPCK1-specific antibody that recognises the cytosolic isoform, whereas PEPCK2 is predominantly a mitochondrial form. Moreover, other important enzymes for gluconeogenic flux such as pyruvate carboxylase and fructose 1,6-bisphosphatase, which are regulated by CREB, HNF $4 \alpha$ and FOXO, might also be affected by PF-4708671. In addition, previous studies have showed that hepatic gluconeogenesis can be regulated 

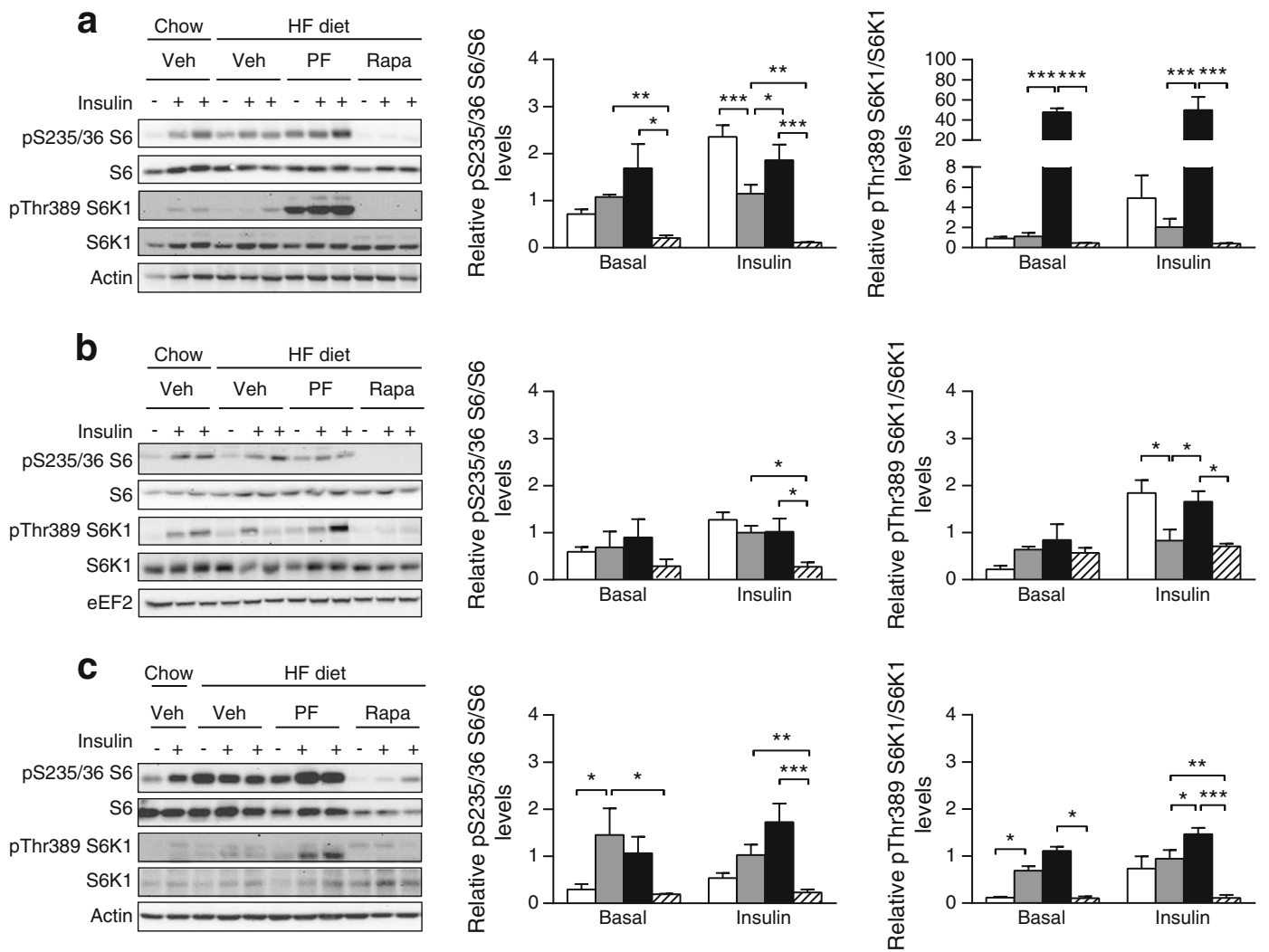

Fig. 7 S6K1 inhibition did not decrease S6 phosphorylation in HF-fed obese mice. Chow-fed mice (white bars), and HF-fed mice treated with vehicle (grey bars), PF-4708671 (black bars) and rapamycin (hatched bars) were fasted for $6 \mathrm{~h}$ before saline or insulin $(3.8 \mathrm{U} / \mathrm{kg}$ caudal, $5 \mathrm{~min}$ ) vein injection and tissues were analysed for S6 and S6K1 phosphorylation. Liver (a), gastrocnemius muscle (b) and epididymal white adipose tissue (c) were lysed and equal amounts of proteins were separated on SDS-PAGE and processed for western blot analyses using the indicated antibodies. $n=5-8$ for each group. ${ }^{*} p<0.05,{ }^{* *} p<0.01$, ${ }^{* * *} p<0.001$. PF, PF-4708671; Rapa, rapamycin; Veh, vehicle independently from the expression of these enzymes. Indeed, changes in gluconeogenic flux have been previously described in humans without changes in PEPCK $1 / 2$ or G6Pase protein/mRNA expression [41]. Moreover, Foretz et al [42] showed that metformin decreases hepatic glucose production via a transcription-independent process, through changes in hepatic energy state, which were also independent from AMPK activation. The detailed molecular mechanisms underlying the suppressive effect of PF-4708671 on hepatic glucose production still need to be fully elucidated.

Whereas PF-4708671 treatment was found to improve glucose tolerance in HF-fed obese mice, the drug failed to improve gluconeogenesis, as measured during the PTT and by determination of the gluconeogenic programme. Nevertheless, other observations suggest that liver insulin sensitivity was improved in PF-4708671-treated obese mice, especially when compared with mice chronically exposed to rapamycin. Indeed, liver Akt S473 phosphorylation was normalised in these mice and liver glycogen content was improved, while hepatic TG levels were decreased in these animals when compared with rapamycin-treated obese mice. Although gluconeogenesis is unaffected, one potential mechanism underlying this hepatic phenotype of PF4708671-treated obese mice may involve a compensatory increase in S6K2 activity. Previous studies have shown that S6K2 can compensate for the loss of $S 6 K 1$ (also known as Rps6kb1) in $S 6 \mathrm{~K} 1 \mathrm{KO}$ mice and that $\mathrm{S} 6 \mathrm{~K} 2$ is the major kinase phosphorylating ribosomal S6 proteins [43, 44]. In mice with partial genetic deletion of both $S 6 K 1$ and $S 6 K 2$ (also known as Rps6kb2) in the liver, suppression of hepatic glucose production by insulin was increased [15], suggesting that S6K2 might also play a role in gluconeogenesis. Indeed, S6K2deficient mice are more insulin sensitive than wild-type mice exposed to HFD [45] - especially given that S6K2 also interacts with Yin Yang 1 [46], a transcriptional factor that promotes hepatic gluconeogenesis [47]. In addition, based on the study of Kim et al [48], S6K2 is more activated in liver of $o b / o b$ mice suggesting that $\mathrm{S} 6 \mathrm{~K} 2$ might also be more active in our HF-diet-induced obesity model. The potential role of compensatory mechanisms after chronic inhibition of S6K1 by PF-4708671 warrants further investigation in future studies.

Current pharmacological treatments fail to achieve optimal glycaemic control and it is therefore critical to develop new therapeutic options for patients with type 2 diabetes. In this 
study, we provide evidence that the specific S6K1 inhibitor PF-4708671 decreased hepatic glucose production and muscle glucose uptake in vitro, and improved glucose tolerance in vivo, which may be of clinical relevance for type 2 diabetes treatment. Indeed, unlike mTORC1/S6K1 inhibition by rapamycin — which has adverse metabolic effects when chronically given in vivo - selective S6K1 inhibition clearly improved glucose tolerance and insulin signalling in metabolic tissues, as revealed by improvement of Akt phosphorylation in HF-fed obese mice. Our results thus suggest that pharmacological inhibition of S6K1 is a valid therapeutic approach for treatment of obesity-linked type 2 diabetes.

Acknowledgements The authors acknowledge V. Dumais, C. Dallaire and C. Dion for their technical help at Cardiology Axis of the Institut Universitaire de Cardiologie et de Pneumologie de Québec (CRIUCPQ), Hôpital Laval, Québec, Canada.

Funding This work was funded by a grant from Canadian Institutes of Health Research (CIHR) to AM (FRN- 57878); MS was supported by a $\mathrm{PhD}$ studentship from Fond de recherche du Québec en santé (FRQS). AM holds a CIHR/Pfizer research Chair on the pathogenesis of insulin resistance and cardiovascular diseases.

Duality of interest The authors declare that there is no duality of interest associated with this manuscript.

Contribution statement All authors were responsible for the conception and design of the study, for the analysis and interpretation of data, and for writing and editing the manuscript, and thus all contributed to its intellectual content. All authors gave final approval of the version to be published. AM is the guarantor of this work and, as such, had full access to all the data in the study and takes responsibility for the integrity of the data and the accuracy of the data analysis.

\section{References}

1. Draznin B (2006) Molecular mechanisms of insulin resistance: serine phosphorylation of insulin receptor substrate-1 and increased expression of p85alpha: the two sides of a coin. Diabetes 55:2392-2397

2. Gual P, Le Marchand-Brustel Y, Tanti JF (2005) Positive and negative regulation of insulin signaling through IRS-1 phosphorylation. Biochimie 87:99-109

3. Fenton TR, Gout IT (2011) Functions and regulation of the $70 \mathrm{kDa}$ ribosomal S6 kinases. Int J Biochem Cell Biol 43:47-59

4. Magnuson B, Ekim B, Fingar DC (2012) Regulation and function of ribosomal protein $\mathrm{S} 6$ kinase (S6K) within $\mathrm{mTOR}$ signalling networks. Biochem J 441:1-21

5. Pardo OE, Seck1 MJ (2013) S6K2: the neglected S6 kinase family member. Front Oncol 3:191

6. Copps KD, White MF (2012) Regulation of insulin sensitivity by serine/threonine phosphorylation of insulin receptor substrate proteins IRS1 and IRS2. Diabetologia 55:2565-2582

7. Hancer NJ, Qiu W, Cherella C, Li Y, Copps KD, White MF (2014) Insulin and metabolic stress stimulate multisite serine/threonine phosphorylation of insulin receptor substrate 1 and inhibit tyrosine phosphorylation. J Biol Chem 289:12467-12484

8. Shah OJ, Hunter T (2006) Turnover of the active fraction of IRS1 involves raptor-mTOR- and S6K1-dependent serine phosphorylation in cell culture models of tuberous sclerosis. Mol Cell Biol 26:6425-6434

9. Veilleux A, Houde VP, Bellmann K, Marette A (2010) Chronic inhibition of the mTORC1/S6K1 pathway increases insulininduced PI3K activity but inhibits Akt 2 and glucose transport stimulation in 3T3-L1 adipocytes. Mol Endocrinol 24:766-778

10. Tremblay F, Brule S, Hee Um S et al (2007) Identification of IRS-1 Ser-1101 as a target of S6K1 in nutrient- and obesity-induced insulin resistance. Proc Natl Acad Sci U S A 104:14056-14061

11. Liu P, Gan W, Inuzuka H et al (2013) Sin1 phosphorylation impairs mTORC2 complex integrity and inhibits downstream Akt signalling to suppress tumorigenesis. Nat Cell Biol 15:1340-1350

12. Um SH, Frigerio F, Watanabe M et al (2004) Absence of S6K1 protects against age- and diet-induced obesity while enhancing insulin sensitivity. Nature 431:200-205

13. Pende M, Kozma SC, Jaquet $M$ et al (2000) Hypoinsulinaemia, glucose intolerance and diminished beta-cell size in S6K1deficient mice. Nature 408:994-997

14. Younis HS, Hirakawa B, Scott W et al (2011) Antisense inhibition of S6 kinase 1 produces improved glucose tolerance and is well tolerated for 4 weeks of treatment in rats. Pharmacology 87:11-23

15. Bae EJ, Xu J, Oh DY et al (2012) Liver-specific p70 S6 kinase depletion protects against hepatic steatosis and systemic insulin resistance. J Biol Chem 287:18769-18780

16. Tremblay F, Marette A (2001) Amino acid and insulin signaling via the $\mathrm{mTOR} / \mathrm{p} 70$ S6 kinase pathway. A negative feedback mechanism leading to insulin resistance in skeletal muscle cells. J Biol Chem 276:38052-38060

17. Tremblay F, Gagnon A, Veilleux A, Sorisky A, Marette A (2005) Activation of the mammalian target of rapamycin pathway acutely inhibits insulin signaling to Akt and glucose transport in 3T3-L1 and human adipocytes. Endocrinology 146:1328-1337

18. Tzatsos A, Kandror KV (2006) Nutrients suppress phosphatidylinositol 3-kinase/Akt signaling via raptor-dependent mTOR-mediated insulin receptor substrate 1 phosphorylation. Mol Cell Biol 26:63-76

19. Houde VP, Brule S, Festuccia WT et al (2010) Chronic rapamycin treatment causes glucose intolerance and hyperlipidemia by upregulating hepatic gluconeogenesis and impairing lipid deposition in adipose tissue. Diabetes 59:1338-1348

20. Deblon N, Bourgoin L, Veyrat-Durebex C et al (2012) Chronic mTOR inhibition by rapamycin induces muscle insulin resistance despite weight loss in rats. Br J Pharmacol 165:2325-2340

21. Pearce LR, Alton GR, Richter DT et al (2010) Characterization of PF-4708671, a novel and highly specific inhibitor of p70 ribosomal S6 kinase (S6K1). Biochem J 431:245-255

22. Bedard S, Marcotte B, Marette A (1997) Cytokines modulate glucose transport in skeletal muscle by inducing the expression of inducible nitric oxide synthase. Biochem J 325(Pt 2):487-493

23. Smadja-Lamere N, Shum M, Deleris P, Roux PP, Abe J, Marette A (2013) Insulin activates RSK (p90 ribosomal S6 kinase) to trigger a new negative feedback loop that regulates insulin signaling for glucose metabolism. J Biol Chem 288:31165-31176

24. Folch J, Lees M, Sloane Stanley GH (1957) A simple method for the isolation and purification of total lipides from animal tissues. J Biol Chem 226:497-509

25. Lo S, Russell JC, Taylor AW (1970) Determination of glycogen in small tissue samples. J Appl Physiol 28:234-236

26. Rosner M, Schipany K, Hengstschlager M (2012) p70 S6K1 nuclear localization depends on its mTOR-mediated phosphorylation at T389, but not on its kinase activity towards S6. Amino Acids 42:2251-2256

27. Vainer GW, Saada A, Kania-Almog J, Amartely A, Bar-Tana J, Hertz R (2014) PF-4708671 activates AMPK independently of p70S6K1 inhibition. PLoS One 9:e107364 
28. Fang Y, Westbrook R, Hill C et al (2013) Duration of rapamycin treatment has differential effects on metabolism in mice. Cell Metab $17: 456-462$

29. Lamming DW, Ye L, Katajisto P et al (2012) Rapamycin-induced insulin resistance is mediated by mTORC2 loss and uncoupled from longevity. Science 335:1638-1643

30. Johnston O, Rose CL, Webster AC, Gill JS (2008) Sirolimus is associated with new-onset diabetes in kidney transplant recipients. Clin J Am Soc Nephrol 19:1411-1418

31. Laplante M, Sabatini DM (2012) mTOR signaling in growth control and disease. Cell 149:274-293

32. Sarbassov DD, Ali SM, Sengupta S et al (2006) Prolonged rapamycin treatment inhibits mTORC2 assembly and Akt/PKB. Mol Cell 22:159-168

33. Ye L, Varamini B, Lamming DW, Sabatini DM, Baur JA (2012) Rapamycin has a biphasic effect on insulin sensitivity in $\mathrm{C} 2 \mathrm{C} 12$ myotubes due to sequential disruption of mTORC1 and mTORC2. Front Genet 3:177

34. Jacinto E, Facchinetti V, Liu D et al (2006) SIN1/MIP1 maintains rictor-mTOR complex integrity and regulates Akt phosphorylation and substrate specificity. Cell 127:125-137

35. Sarbassov DD, Guertin DA, Ali SM, Sabatini DM (2005) Phosphorylation and regulation of Akt/PKB by the rictor-mTOR complex. Science 307:1098-1101

36. Liu P, Guo J, Gan W, Wei W (2014) Dual phosphorylation of Sin 1 at T86 and T398 negatively regulates mTORC2 complex integrity and activity. Protein Cell 5:171-177

37. Di R, Wu X, Chang Z et al (2012) S6K inhibition renders cardiac protection against myocardial infarction through PDK1 phosphorylation of Akt. Biochem J 441:199-207

38. Mendez-Lucas A, Duarte JA, Sunny NE et al (2013) PEPCK-M expression in mouse liver potentiates, not replaces, PEPCK-C mediated gluconeogenesis. J Hepatol 59:105-113

39. Stark R, Guebre-Egziabher F, Zhao X et al (2014) A role for mitochondrial phosphoenolpyruvate carboxykinase (PEPCK-M) in the regulation of hepatic gluconeogenesis. J Biol Chem 289: 7257-7263

40. van Schaftingen E, Gerin I (2002) The glucose-6-phosphatase system. Biochem J 362:513-532

41. Samuel VT, Beddow SA, Iwasaki T et al (2009) Fasting hyperglycemia is not associated with increased expression of PEPCK or G6Pc in patients with type 2 diabetes. Proc Natl Acad Sci U S A 106:12121-12126

42. Foretz M, Hebrard S, Leclerc J et al (2010) Metformin inhibits hepatic gluconeogenesis in mice independently of the LKB1/AMPK pathway via a decrease in hepatic energy state. $\mathrm{J}$ Clin Invest 120:2355-2369

43. Pende M, Um SH, Mieulet V et al (2004) S6K1(-/-)/S6K2(-/-) mice exhibit perinatal lethality and rapamycin-sensitive 5'-terminal oligopyrimidine mRNA translation and reveal a mitogen-activated protein kinase-dependent S6 kinase pathway. Mol Cell Biol 24:3112-3124

44. Shima H, Pende M, Chen Y, Fumagalli S, Thomas G, Kozma SC (1998) Disruption of the $\mathrm{p} 70(\mathrm{~s} 6 \mathrm{k}) / \mathrm{p} 85(\mathrm{~s} 6 \mathrm{k})$ gene reveals a small mouse phenotype and a new functional S6 kinase. EMBO J 17:6649-6659

45. Taylor KM, Bajko J, Cabrera MS et al (2014) S6 kinase 2 deficiency improves glucose disposal in mice fed a high fat diet. J Diabetes Metab 5:441. doi:10.4172/2155-6156.1000441

46. Ismail HM, Myronova O, Tsuchiya Y, Niewiarowski A, Tsaneva I, Gout I (2013) Identification of the general transcription factor Yin Yang 1 as a novel and specific binding partner for S6 kinase 2. Cell Signal 25:1054-1063

47. Lu Y, Xiong X, Wang X et al (2013) Yin Yang 1 promotes hepatic gluconeogenesis through upregulation of glucocorticoid receptor. Diabetes 62:1064-1073

48. Kim K, Pyo S, Um SH (2012) S6 kinase 2 deficiency enhances ketone body production and increases peroxisome proliferatoractivated receptor alpha activity in the liver. Hepatology 55:1727-1737 